\title{
In vitro investigation of antifungal activity of allicin alone and in combination with azoles against Candida species.
}

\begin{abstract}
Candidiasis is a term describing infections by yeasts from the genus Candida, and the type of infection encompassed by candidiasis ranges from superficial to systemic. Treatment of such infections often requires antifungals such as the azoles, but increased use of these drugs has led to selection of yeasts with increased resistance to these drugs. In this study, we used allicin, an allyl sulfur derivative of garlic, to demonstrate both its intrinsic antifungal activity and its synergy with the azoles, in the treatment of these yeasts in vitro. In this study, the MIC50 and MIC90 of allicin alone against six Candida spp. ranged from 0.05 to $25 \mu \mathrm{g} / \mathrm{ml}$. However, when allicin was used in combination with fluconazole or ketoconazole, the MICs were decreased in some isolates. Our results demonstrated the existing synergistic effect between allicin and azoles in some of the Candida spp. such as C. albicans, C. glabrata and C. tropicalis, but synergy was not demonstrated in the majority of Candida spp. tested. Nonetheless, In vivo testing needs to be performed to support these findings.
\end{abstract}

Keyword: Allicin; Azoles; Candida species; MIC. 